\title{
Attenuation of Neural Responses in Primary Visual Cortex during the Attentional Blink
}

\author{
Mark A. Williams, ${ }^{1}$ Troy A. W. Visser, ${ }^{2}$ Ross Cunnington, ${ }^{3,4}$ and Jason B. Mattingley ${ }^{3,4}$ \\ ${ }^{1}$ Macquarie Centre for Cognitive Science, Macquarie University, Sydney, New South Wales 2109, Australia, ${ }^{2}$ Department of Psychology, University of British \\ Columbia Okanagan, Kelowna, British Columbia, Canada V1V 1V7, and ${ }^{3}$ Queensland Brain Institute and ${ }^{4}$ School of Psychology, University of Queensland, \\ St. Lucia, Queensland 4072, Australia
}

Information-processing bottlenecks are characteristic of many cognitive and neural systems. One such bottleneck is revealed by tasks in which rapidly successive stimulus events must be reported. Here, observers missed the second of two visual targets if it occurred within $700 \mathrm{~ms}$ of the first [an "attentional blink" $(\mathrm{AB})$ ], even though this second target could be reported accurately when the first item was ignored. Isolating neural responses to such rapid events has proven difficult because current magnetic resonance imaging methods rely on relatively sluggish changes in the brain's physiological response to sensory inputs. Here, we overcame this limitation by presenting successive visual targets at different spatial locations, thereby exploiting the retinotopic organization of early cortical visual areas to distinguish neural activity associated with successive target events. We show that neural activity in primary visual cortex is significantly modulated during the $\mathrm{AB}$, and that this activity mirrors behavioral measures of target identification accuracy. The findings suggest that the neural signature of perceptual suppression during processing of rapidly successive stimuli is evident at the earliest stages of cortical sensory processing.

Key words: attention; attentional blink; temporal selection; fMRI; vision; human

\section{Introduction}

Information-processing bottlenecks are a prominent feature in many cognitive systems. One such capacity limitation can be seen in visual cueing paradigms in which cues that accurately predict a target location typically facilitate target response times and accuracy (Posner, 1980). This suggests that processing is benefited when limited resources (i.e., attention) can be allocated to a target location before its occurrence. An analogous situation arises when two targets are presented at the same location within $\sim 500$ ms. Here, second-target (T2) accuracy is significantly impaired [the "attentional blink" (AB)] (Raymond et al. 1992; Visser, 2007), implying that allocation of capacity-limited resources to the first target (T1) prevents complete processing of T2.

A significant volume of neurophysiological research has been devoted to examining the underpinnings of capacity limitations in vision. In the case of spatial attention, evidence from visual cueing paradigms suggests that behavioral effects are reflected in neural activity as early as primary visual cortex (V1) (Hopfinger and Mangun, 1998; Hopfinger et al., 2000; McMains and Somers, 2004). In contrast, in the case of temporal attention, evidence from $\mathrm{AB}$ experiments has implicated later cognitive mechanisms, such as working memory and spatial selection (Vogel et al., 1998;

Received July 1, 2008; accepted August 20, 2008.

This work was supported by a grant from Unilever Research, UK. M.A.W. is a C.J. Martin Fellow sponsored by the Australian National Health and Medical Research Council. We thank Chris Baker, Chris Chambers, Adam Morris, Anina Rich, and Ed Vul for their comments and suggestions on this work.

Correspondence should be addressed to Mark A. Williams, Macquarie Centre for Cognitive Science, Macquarie University, Sydney, New South Wales 2109, Australia. E-mail: mark.williams@maccs.mq.edu.au.

D0I:10.1523/JNEUROSCI.3057-08.2008

Copyright $\odot 2008$ Society for Neuroscience $\quad$ 0270-6474/08/289890-05\$15.00/0
Marois et al., 2000, 2004; Marcantoni et al., 2003; Kranczioch et al., 2005, 2007; Jolicoeur et al., 2006; Martens et al., 2006; Johnston et al., 2007; Shapiro et al., 2007).

Although past studies of the $A B$ have not examined whether there is a link between early visual mechanisms and the $A B$, there are several reasons to believe such a link might exist. First, parietal neglect patients, who have difficulty allocating attention to the contralesional side of space, also show a prolonged $\mathrm{AB}$ for sequential displays at the fovea (Husain et al. 1997; Van Vleet and Robertson, 2006), implying a functional overlap between the two deficits that may extend to early visual areas. Second, studies of the primate visual system suggest that modulations of early visual processing (Hupé et al., 1998) are instantiated by extensive feedback projections from extrastriate regions of the temporal and parietal cortex to the V1 (Noesselt et al., 2002; Weidner et al., 2006; Hinrichs et al., 2008). In light of the demonstrable influence of the $\mathrm{AB}$ on activity in temporal and parietal cortices (Marois and Ivanoff, 2005), such feedback projections might also be expected to lead to differences in early visual activity. Finally, several studies have shown that adapting light levels influence the AB (Maki and Padmanabhan, 1994; Giesbrecht et al., 2004), implicating low-level visual processes.

In the present work, we used functional magnetic resonance imaging (fMRI) to determine whether T2-related activity in early visual areas is modulated during the AB. Previous fMRI investigations have been limited in their ability to detect T2-related activity because conventional paradigms present all items from the same, typically foveal, spatial location. Thus, the relatively slow hemodynamic response to $\mathrm{T} 1$ overlaps with the response to T2. To overcome this problem, we presented T1 at the fovea, and 


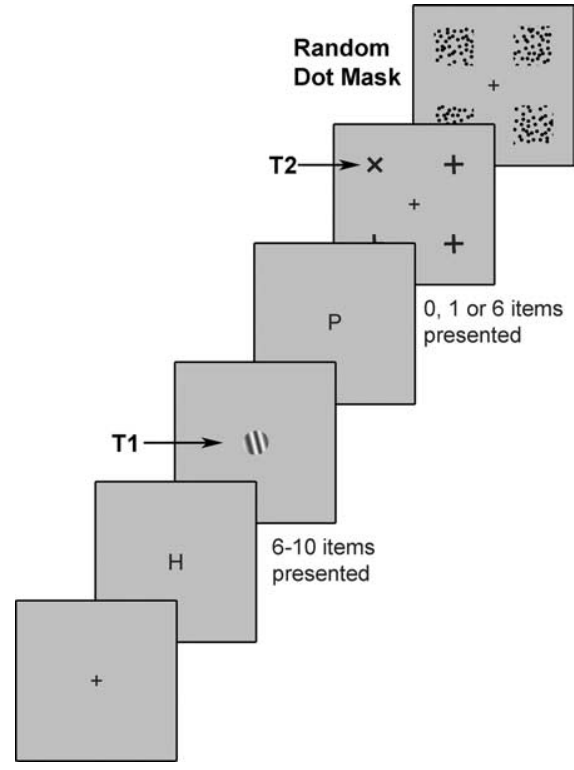

Figure 1. Schematic of the sequence of events in the attentional blink task. Each stimulus display was presented for $17 \mathrm{~ms}$, followed by a blank interval of $83 \mathrm{~ms}$. Trials commenced with a central fixation cross for $1000 \mathrm{~ms}$. This was followed immediately by $6-10$ central letters (distractors) before the appearance of the first target ( $\mathrm{T} 1$, a square-wave grating tilted $15^{\circ}$ clockwise or anticlockwise from vertical). After $\mathrm{T1}$, a variable number of additional letters appeared $(0,1$, or 6 items, corresponding to T1-T2 lags of 100,200 , or $700 \mathrm{~ms})$. The second target display (T2) consisted of three + symbols and a single $x$, each located $4.2^{\circ}$ from fixation in one of the four quadrants of the visual field. Each item in the T2 display was masked by a patch of random-dot motion $(83 \mathrm{~ms})$ in each of the four quadrants. Subjects indicated the location of the $x$ via a button press at the end of the trial.

T2 at one of four peripheral locations. This allowed us to take advantage of the retinotopic organization of early visual cortical areas to separate neural activity associated with each target (Horton and Hoyt, 1991; McMains and Somers, 2004).

\section{Materials and Methods}

Subjects. We initially tested 12 observers in the AB task outside of the MRI scanner. This pilot study was designed to identify individuals who showed a substantial $\mathrm{AB}$, defined as a reduction in $\mathrm{T} 2$ accuracy of $15 \%$ or more at either of the two shortest T1-T2 asynchronies relative to the longest asynchrony (see supplemental Fig. 1, available at www. jneurosci.org as supplemental material). The five observers selected for scanning (three male; two female) had a mean age of 29.4 years $(\mathrm{SD}=7)$. They all had normal or corrected-to-normal vision and gave informed consent to participate.

Method. A sample experimental trial is illustrated in Figure 1. Unless otherwise stated, all stimuli subtended $\sim 0.6^{\circ} \times 0.6^{\circ}$, were presented for $17 \mathrm{~ms}$, and were separated from the next item by an $83 \mathrm{~ms}$ interval during which the display was blank. These rapid stimulus presentation rates are similar to those used in conventional $\mathrm{AB}$ experiments and are designed to limit the processing time available for each item (Raymond et al., 1992). Each trial began with the presentation of a central fixation cross for 1000 $\mathrm{ms}$. This was followed by the central presentation of 6-10 letters chosen randomly without replacement from all letters of the English alphabet, and then T1, which was a square-wave grating tilted $15^{\circ}$ clockwise or anticlockwise from vertical. The stimulus onset asynchrony (SOA) between the first and second targets was either 100, 200, or $700 \mathrm{~ms}$. At the $100 \mathrm{~ms}$ SOA, targets followed one another directly; at the $200 \mathrm{~ms} \mathrm{SOA}$, there was a single intervening distractor, and at the $700 \mathrm{~ms} \mathrm{SOA}$, there were six intervening distractors. The SOA was chosen randomly on each trial with the constraint that each SOA occurred equally often in the experiment. The second target consisted of an $\mathrm{X}$, embedded within a patch $\left(1.6^{\circ} \times 1.6^{\circ}\right)$ of random-dot motion, and presented with equal probability at one of four peripheral locations $4.2^{\circ}$ from fixation. This target was accompanied by + signs, also embedded in random-dot motion patches, presented at the remaining three peripheral locations. To mask T2, random-dot motion was presented at all four possible T2 locations for $83 \mathrm{~ms}$. At the end of each trial, subjects indicated the orientation of the T1 grating, and the location of $\mathrm{T} 2$ by pressing one of two keys on a button box. This initiated the next trial.

In the experimental condition, which was divided into two runs, subjects completed 198 experimental trials in which they identified both T1 and T2. In a separate control condition consisting of a further 198 trials, divided into two runs, the identical stimuli were presented but subjects were instructed to ignore $\mathrm{T} 1$ and report only the location of T2.

In addition to experimental and control trials, we also conducted separate localizer scans consisting of passive viewing of $16 \mathrm{~s}$ blocks of central stream items with intermittent T1 presentations $(N=6), 16 \mathrm{~s}$ blocks of the four random-dot motion displays with intermittent $\mathrm{T} 2$ presentations $(N=6)$, and 16 s blocks of the fixation cross alone as a baseline condition $(N=6)$. This procedure allowed us to localize representations in $\mathrm{V} 1$ that corresponded to the four peripheral T2 stimuli (crosses plus random-dot motion). To ensure that our analyses incorporated the primary visual cortex (V1), we determined the four peak activities that corresponded to the T2 locations and applied $5 \mathrm{~mm}$ diameter masks along the edge of the calcarine sulcus (Dougherty et al., 2003) (Fig. 2).

Functional magnetic resonance images were acquired using a wholebody 1.5 tesla Siemens MAGNETOM Avanto scanner with a gradientecho echo-planar imaging (EPI) sequence. The scanner was equipped with a standard radiofrequency birdcage headcoil for signal transmission and reception. Lateral head stabilizers were used to minimize head movement. EPI images were acquired at a $30^{\circ}$ axial oblique plane using a gradient-echo pulse sequence and interleaved slice acquisition [repetition time (TR), $1.5 \mathrm{~s}$; echo time, $40 \mathrm{~ms}$; flip angle, $90^{\circ}$; 16 contiguous slices with a resolution of $1.7 \times 1.7 \times 4.0 \mathrm{~mm}$, without an interslice gap, and with an in-plane resolution of $128 \times 120]$. Each functional run began with eight dummy TRs, to allow for steady-state tissue magnetization. High-resolution, magnetization-prepared rapid-acquisition gradient echo structural images were also obtained for each subject.

During fMRI acquisition, the images were back-projected via a Sony SVGA LCD data projector (VPL-SC1) onto an opaque screen positioned beyond the observers' feet. Subjects viewed the screen through a mirror mounted on the headcoil. Customized software written in Visual Basic 6.0, and using DirectX 9.0b technology (Microsoft Corporation), was used to present the stimuli. Optic-fiber button boxes were used to record subject responses.

\section{Results}

\section{Behavioral}

As can be seen in Figure $3 A$, in the control (single-task) condition, T2 identification was uniformly high and did not vary as a function of T1-T2 SOA $(p>0.40)$. In contrast, in the experimental (dual-task) condition, T2 identification varied as a function of T1-T2 SOA; accuracy at the $200 \mathrm{~ms}$ SOA was significantly lower than that at SOAs of 100 and $700 \mathrm{~ms}, F_{(2,8)}=28.01, p<$ 0.001 . This pattern of results represents the behavioral signature of the AB deficit (Visser et al., 1999). To confirm the reliability of our results, we also examined data from each subject separately. As can be seen in Figure $3 C$, each subject showed a robust $A B$ deficit.

\section{Imaging}

Data were processed and analyzed using SPM2 (Wellcome Department of Imaging Neuroscience, Institute of Neurology, London, UK; http://www.fil.ion.ucl.ac.uk/spm). For each subject, the EPI images were realigned to the first image for movement correction using a least-squared approach and six-parameter rigid body spatial transformations. The EPI images from the localizer runs were spatially smoothed using an isotropic Gaussian kernel at $6 \mathrm{~mm}$ full-width half-maximum. The effects of the localizer paradigm were estimated for each subject on a voxel-by-voxel basis using the principles of the general linear model. These data 


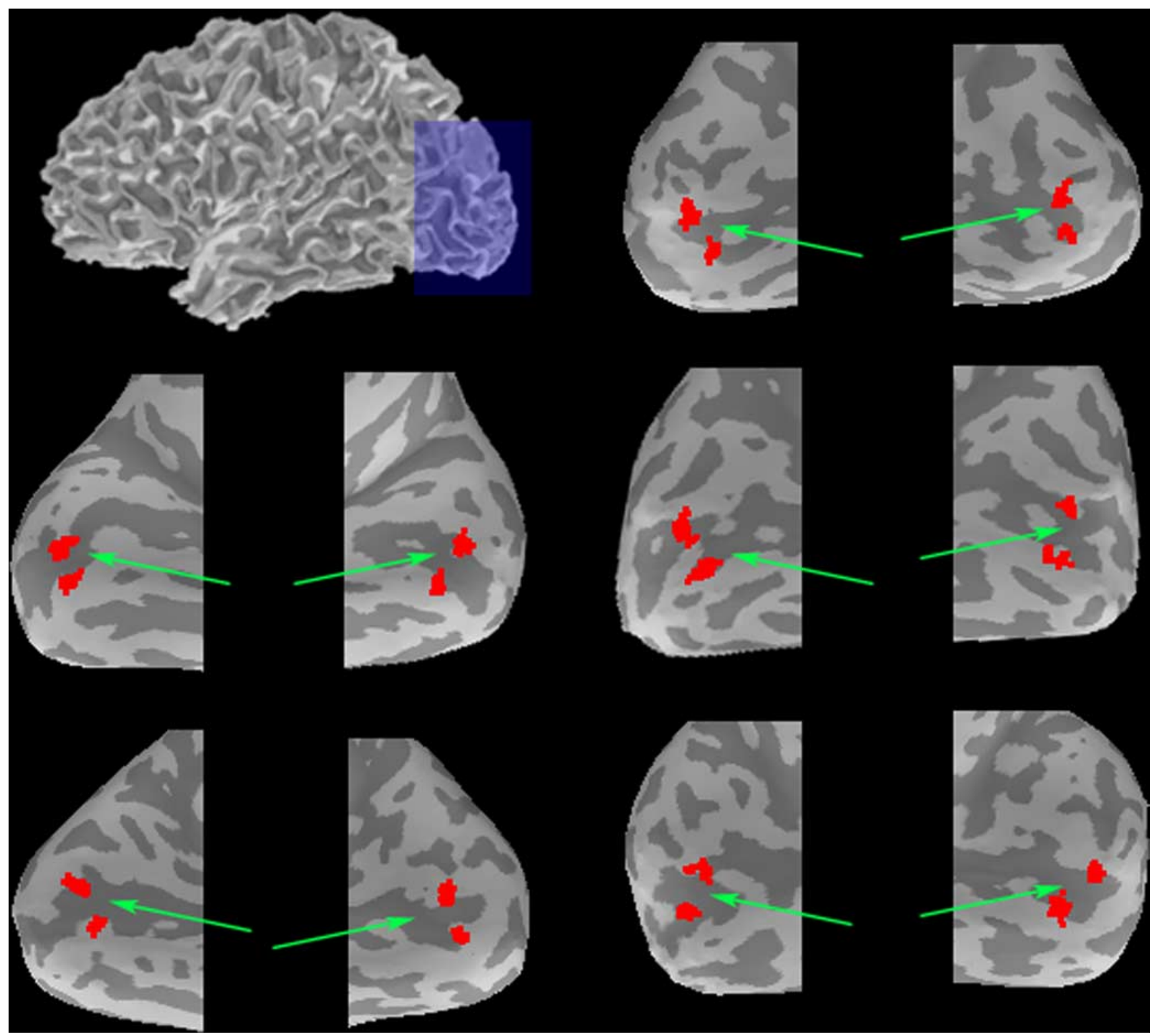

Figure 2. Functionally defined ROIs used for the analysis of activation associated with T2 events in the single- versus dual-task versions of the attentional blink paradigm. ROls (shown in red) are superimposed on inflated left and right hemispheres for each of the five subjects in the fMRI study. The green arrows point to the calcarine sulcus.

were analyzed using boxcar functions convolved with the canonical hemodynamic response function after low-frequency noise was removed with $128 \mathrm{~ms}$ high-pass filtering. Data from the main experimental task were not spatially smoothed. The percentage signal change was extracted from each of the regions of interest acquired from the independent localizer scans, and was subjected to an ANOVA.

As can be seen in Figure $3 B$, the $A B$ effect observed in the behavioral data were mirrored by changes in the blood oxygen level-dependent (BOLD) signal within the four regions of interest (ROIs) identified independently from the localizer scans, and centered on the primary visual cortex, $F_{(2,8)}=4.98, p<0.05$. Separate examination of individual data showed that this correspondence between behavioral performance and neural activity was apparent for each of our five subjects (Fig. $3 C, D$ ). Together, the behavioral and imaging results suggest that the decrement in $\mathrm{T} 2$ identification observed during the $\mathrm{AB}$ is associated with significant attenuation of neural activity in primary visual cortex.

\section{Discussion}

The present study examined the neural correlates of the AB deficit, a striking impairment in second-target accuracy that occurs whenever two targets must be identified within approximately half a second of each other. This second-target deficit has conventionally been attributed to capacity limitations: specifically, that attention cannot be allocated to a second target while it is still occupied with the first. To examine the role of early visual processing in the $\mathrm{AB}$, we used a modified paradigm in which targets were presented at different locations on the retina. This allowed us to show for the first time that capacity limitations implicated in the $\mathrm{AB}$ deficit can modulate processing as early as primary visual cortex.

Two notable, and related, aspects of the data deserve additional comment. First, we found that accuracy at the shortest T1-T2 SOA, when T2 followed T1 directly, was better than when the targets were separated by a single distractor $(\mathrm{T} 1-\mathrm{T} 2 \mathrm{SOA}=$ 
A)

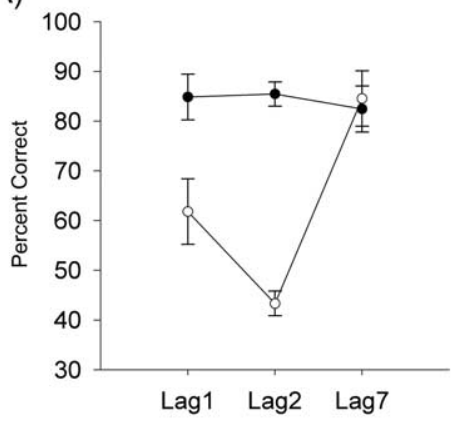

C)

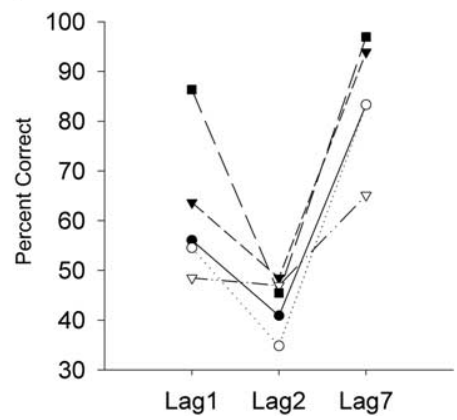

B)

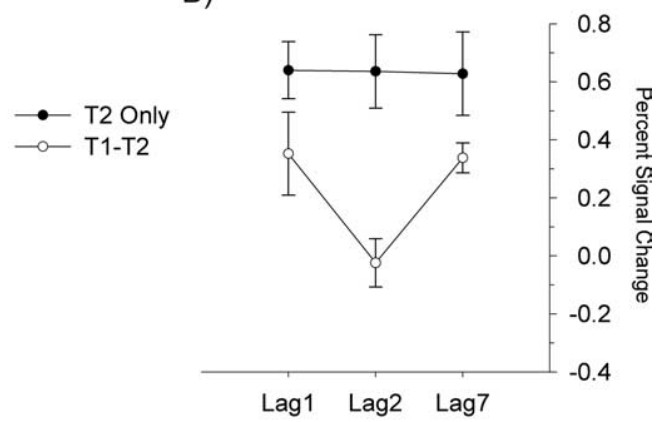

D)

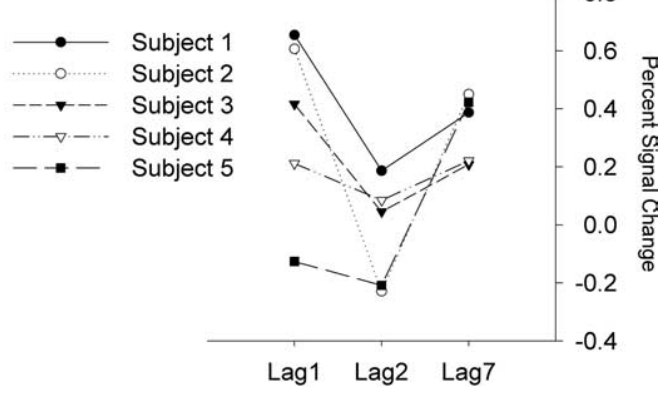

Figure 3. Behavioral data and percentage BOLD signal change $( \pm S E)$ for the group and for each of the five individual subjects. $A$, Mean percent correct responses for the T2-only task, in which subjects were instructed to ignore the appearance of T1, and for the T1-T2 task, in which subjects had to report both the orientation of the T1 grating and the quadrant within which the T2 cross appeared. B, Mean BOLD responses for the four early cortical ROls, as defined by the independent localizer scans. $\boldsymbol{C}$, Percent correct responses for each of the five individual subjects, showing performance for the T2-only and T1-T2 tasks. D, Mean BOLD responses for the four early cortical ROls for each individual subject.

$200 \mathrm{~ms}$ ). This pattern of results, conventionally termed "lag-1 sparing” (Potter et al., 1998; Visser et al., 1999), has been explained by suggesting that when T2 appears immediately after T1, it is able to enter the attentional gate triggered by T1, and thus receives limited-capacity resources. Lag-1 sparing is relatively common, having been found in approximately one-half of all published studies on the AB deficit (Visser et al., 1999). What is surprising, however, is that we found this pattern of results when $\mathrm{T} 1$ and T2 events appeared in different spatial locations: conditions that have almost invariably failed to yield lag-1 sparing in previous investigations (Visser et al., 1999). An important clue to why we obtained lag-1 sparing here is suggested by Jefferies et al. (2007), who showed that when there is spatial uncertainty associated with $\mathrm{T} 1$ and $\mathrm{T} 2$ events, subjects divide their attention across possible target locations, thus allowing both targets to gain access to the same attentional gate.

A second notable finding is that the presence of lag-1 sparing, in both the behavioral and imaging data, rules out an obvious alternative explanation for our pattern of results. Specifically, it might have been argued that the attenuation in BOLD signal intensity we observed at the $200 \mathrm{~ms}$ T1-T2 SOA simply reflects the time required by observers to shift their spatial attention from $\mathrm{T} 1$ at fixation to the peripheral location at which $\mathrm{T} 2$ appeared. If this account were correct, however, visual cortex activity associated with T2 stimuli should have increased as the SOA between $\mathrm{T} 1$ and $\mathrm{T} 2$ became larger. Instead, mirroring the pattern of lag-1 sparing found behaviorally, the BOLD signal within the V1 ROIs was significantly smaller at $200 \mathrm{~ms}$ versus $100 \mathrm{~ms}$ SOA.

A third aspect of our data that deserves comment concerns the spatial extent of the capacity limitations elicited by $\mathrm{T} 1$ processing.
That suppression of early visual processing occurred for T2 despite it being separated from $\mathrm{T} 1$ by nearly $5^{\circ}$ of visual angle, implies that the deficits arising from T1 processing are not limited to the spatial location of T1. To examine the spatial extent of visual suppression further, we compared the magnitude of the BOLD response within the ROI corresponding to the quadrant in which the target cross (X) appeared with the magnitude of the response for the ROIs associated with the distractors $(+)$ across the different SOAs. This twoway ANOVA yielded no significant interaction between ROI and SOA ( $p>0.10)$, and no main effect of ROI $(p>0.10)$. Crucially, however, the main effect of SOA was significant, $F_{(3,12)}=5.00, p<0.05$, suggesting that the decrease in visual cortex activity at shorter SOAs occurred across the entire visual field. This is consistent with the findings of Kristjánsson and Nakayama (2002), who showed a robust $\mathrm{AB}$ for $\mathrm{T} 2$ events even when these were separated from $\mathrm{T} 1$ by $15^{\circ}$ of visual angle. Our results are also reminiscent of those from the "inhibition of return" literature, which shows that inhibition extends to spatial locations adjacent to nonpredictive cues (Bennett and Pratt, 2001). The similarity between findings arising from these different studies is intriguing and clearly deserves further investigation.

A final issue for discussion concerns the relationship between our findings and those of previous fMRI studies. Whereas previous $\mathrm{AB}$ studies have shown reliable modulation of $\mathrm{T} 2$ processing in temporal, frontal, and parietal regions of extrastriate cortex (Marois and Ivanoff, 2005), the present work is the first to find similar modulations in the primary visual cortex. We suggest that a theoretical framework for unifying all of these studies can be found in the notion of reentrant pathways (Mumford, 1992). As noted earlier, feedback projections are a ubiquitous feature of the primate visual processing system. A functional role for these projections in the AB has been suggested by Giesbrecht and Di Lollo (1998) (see also Di Lollo et al., 2000). They proposed that object identification depends on the establishment of iterative feedback loops between higher and lower visual areas that allow "hypotheses" about object identities generated by higher areas to be tested via comparisons with visual feature information available in early areas. On this account, the $\mathrm{AB}$ arises because $\mathrm{T} 1$ processing prevents the iterative feedback process required for $\mathrm{T} 2$ identification from being completed before masking of $\mathrm{T} 2$ by the item presented immediately after it.

This account of the $\mathrm{AB}$, based on the joint activity of extrastriate and striate visual areas, explains why activity changes in both of these areas might be found during the $\mathrm{AB}$. It also predicts that modulations of neural activity in early visual areas will not necessarily be found during the initial feedforward sweep of visual processing but, instead, are more likely to occur later in processing as iterative loops are established. This prediction is consistent with earlier failures to find modulations of early components during the $A B$ (Vogel et al., 1998). It also suggests a possible alternative interpretation of $\mathrm{N} 2 \mathrm{pc}$ modulations during the $\mathrm{AB}$ 
recently described by Jolicoeur et al. (2006). Such modulations may not merely reflect attentional selection, but may also imply modulations in iterative processing.

Finally, an iterative account of the present results, which emphasizes the relationship between neural activity in extrastriate and striate visual areas, parallels hypotheses advanced to explain modulations of V1 activity in spatial attention tasks. For example, Luck et al. (1997) and Rees et al. (1997) have all shown evidence consistent with the notion that variations in V1 activity reflect biasing signals from later, extrastriate visual areas. It is also notable that patients with visual neglect reliably display prolonged $\mathrm{AB}$ deficits (Husain et al. 1997; Van Vleet and Robertson, 2006), suggesting a further link between spatial and temporal attention. Such parallels between theoretical and empirical considerations demonstrate the importance of examining links between capacity limitations across a variety of behavioral paradigms to better understand how such bottlenecks affect human perception. Our findings suggest that it is vital to consider the influence of feedback projections in theoretical accounts of the $\mathrm{AB}$ and related perceptual phenomena.

\section{References}

Bennett PJ, Pratt J (2001) The spatial distribution of inhibition of return. Psychol Sci 12:76-80.

Di Lollo V, Enns JT, Rensink RA (2000) Competition for consciousness among visual events: the psychophysics of reentrant visual processes. J Exp Psychol Gen 129:481-507.

Dougherty RF, Koch VM, Brewer AA, Fischer B, Modersitzki J, Wandell BA (2003) Visual field representations and locations of visual areas V1/2/3 in human visual cortex. J Vis 3:586-598.

Giesbrecht B, Di Lollo V (1998) Beyond the attentional blink: visual masking by object substitution. J Exp Psychol Hum Percept Perform 24:1454-1466.

Giesbrecht B, Bischof WF, Kingstone A (2004) Seeing the light: adapting luminescence reveals low-level visual processes in the attentional blink. Brain Cognit 55:307-309.

Hinrichs H, Noesselt T, Heinze HJ (2008) Directed information flow: a model free measure to analyze causal interactions in event related EEGMEG-experiments. Hum Brain Mapp 29:193-206.

Hopfinger JB, Mangun GR (1998) Reflexive attention modulates processing of visual stimuli in human extrastriate cortex. Psychol Sci 9:441-447.

Hopfinger JB, Buonocore MH, Mangun GR (2000) The neural mechanisms of top-down attentional control. Nat Neurosci 3:284-291.

Horton JC, Hoyt WF (1991) The representation of the visual field in human striate cortex. A revision of the classic Holmes map. Arch Ophthalmol 109:816-824

Hupé JM, James AC, Payne BR, Lomber SG, Girard P, Bullier J (1998) Cortical feedback improves discrimination between figure and background by V1, V2, and V3 neurons. Nature 394:784-787.

Husain M, Shapiro K, Martin J, Kennard C (1997) Abnormal temporal dynamics of visual attention in spatial neglect patients. Nature 385:154-156.

Jefferies LN, Ghorashi S, Kawahara J, Di Lollo V (2007) Ignorance is bliss: The role of observer expectation in dynamic spatial tuning of the attentional focus. Percept Psychophys 69:1162-1174.

Johnston SJ, Shapiro KL, Vogels W, Roberts NJ (2007) Imaging the attentional blink: perceptual versus attentional limitations. Neuroreport 18:1475-1478.
Jolicoeur P, Sessa P, Dell'Acqua R, Robitaille N (2006) Attentional control and capture in the attentional blink paradigm: evidence from human electrophysiology. Eur J Cognit Psychol 18:560-578.

Kranczioch C, Debener S, Schwarzbach J, Goebel R, Engel AK (2005) Neural correlates of conscious perception in the attentional blink. Neuroimage 24:704-714.

Kranczioch C, Debener S, Maye A, Engel AK (2007) Temporal dynamics of access to consciousness in the attentional blink. Neuroimage 37:947-955.

Kristjánsson A, Nakayama K (2002) The attentional blink in space and time. Vis Res 42:2039-2050.

Luck SJ, Chelazzi L, Hillyard SA, Desimone R (1997) Neural mechanisms of spatial selective attention in areas V1, V2, and V4 of macaque visual cortex. J Neurophysiol 77:24-42.

Maki WS, Padmanabhan G (1994) Transient suppression of processing during rapid serial visual presentation: Acquired distinctiveness of probes modulates the attentional blink. Psychon Bull Rev 1:499-504.

Marcantoni WS, Lepage M, Beaudoin G, Bourgouin P, Richer F (2003) Neural correlates of dual task interference in rapid visual streams: an fMRI study. Brain Cogn 53:318-321.

Marois R, Ivanoff J (2005) Capacity limits of information processing in the brain. Trends Cogn Sci 9:296-305.

Marois R, Chun MM, Gore JC (2000) Neural correlates of the attentional blink. Neuron 28:299-308.

Marois R, Yi DJ, Chun MM (2004) The neural fate of consciously perceived and missed events in the attentional blink. Neuron 41:465-472.

Martens S, Munneke J, Smid H, Johnson A (2006) Quick minds don't blink: electrophysiological correlates of individual differences in attentional selection. J Cogn Neurosci 18:1423-1438.

McMains, SA, Somers DC (2004) Multiple spotlights of attentional selection in human visual cortex. Neuron 42:677-686.

Mumford D (1992) On the computational architecture of the neocortex. Biol Cybern 66:241-251.

Noesselt T, Hillyard SA, Woldorff MG, Schoenfeld A, Hagner T, Jäncke L, Tempelmann C, Hinrichs H, Heinze HJ (2002) Delayed striate cortical activation during spatial attention. Neuron 35:575-587.

Posner MI (1980) Orienting of attention. Q J Exp Psychol 32:3-25.

Potter MC, Chun MM, Banks BS, Muckenhoupt M (1998) Two attentional deficits in serial target search: the visual attentional blink and an amodal task-switch deficit. J Exp Psychol Learn Mem Cogn 24:979-992.

Raymond JE, Shapiro KL, Arnell KM (1992) Temporary suppression of visual processing in an RSVP task: an attentional blink? J Exp Psychol Hum Percept Perform 18:849-860.

Rees G, Frackowiak R, Frith C (1997) Two modulatory effects of attention that mediate object categorization in human cortex. Science 275:835-838.

Shapiro KL, Johnston SJ, Vogels W, Zaman A, Roberts N (2007) Increased functional magnetic resonance imaging activity during nonconscious perception in the attentional blink. Neuroreport 18:341-345.

Van Vleet TM, Robertson LC (2006) Cross-modal interactions in time and space: auditory influence on visual attention in hemispatial neglect. J Cogn Neurosci 18:1368-1379.

Visser T, Bischof WF, Di Lollo V (1999) Attentional switching in spatial and non-spatial domains: evidence from the attentional blink. Psychol Bull 125:458-469.

Vogel EK, Luck SJ, Shapiro KL (1998) Electrophysiological evidence for a postperceptual locus of suppression during the attentional blink. J Exp Psychol Hum Percept Perform 24:1656-1674.

Weidner R, Shah NJ, Fink GR (2006) The neural basis of perceptual hypothesis generation and testing. J Cogn Neurosci 18:258-266. 